Pacific Journal of Mathematic 


\title{
ON INTEGRAL REPRESENTATIONS OF PIECEWISE HOLOMORPHIC FUNCTIONS
}

\author{
GerhaRD K. KALISCH
}

Let $D$ be the interior of the unit circle in $C, D^{c}$ its exterior and $T$ the unit circumference. We consider certain piecewise holomorphic functions that are holomorphic in $D$ and also in $D^{c}$. This paper deals with those piecewise holomorphic functions that are representable by means of complex Poisson-Stieltjes integrals on $T$; we call this set of functions $P_{1}$. The set of all piecewise holomorphic functions (holomorphic in $D$ and in $D^{c}$ ) we call $P$. Earlier work-see Rolf Nevanlinna, Eindeutige Analytische Funktionen, Springer, Berlin, 1953 and references there-dealt with positive (Herglotz-Riesz) or real (Nevanlinna) measures; we shall use here the entire space $M$ of bounded complex Borel measures on $T$. This gives the theory more flexibility. We consider characterizations of functions in $P$ representable by means of complex Poisson-Stieltjes integrals, uniqueness questions, the nature of the mapping between the subset $P_{1}$ of $P$ of representable functions and $M$, as well as the ring structures in $M$ (under convolution) and $P_{1}$ (Hadamard products), and questions of derivatives and integrals. We end with an application to Fourier-Stieltjes moments relative to measues in $M$.

We call a function $F \in P$ representable if there is a measure $m \in M$ so that $F=\int P_{C} d m+k$ where $P_{C}=P_{C}(z)=\left(e^{i t}+z\right) /\left(e^{i t}-z\right)$ is the complex Poisson kernel, $k$ is a piecewise constant function in $P$, and where the limits of integration are omitted when they are 0 and $2 \pi$ respectively. A function $F \in P$ is said to be of real type if $F\left(\bar{z}^{-1}\right)=-\overline{F(z)}$ for all $z \in D \cup D^{c}$. The functions

$$
G=G_{F}(z)=\frac{1}{2}\left(F(z)-\overline{F\left(\bar{z}^{-1}\right)}\right), H=H_{F}(z)=-\frac{1}{2}\left(i F(z)+i \overline{F\left(\bar{z}^{-1}\right)}\right)
$$

are of real type; we have $F=G+i H$ and $F \in P_{1}$ if and only if $G$ and $H$ are in $P_{1}$. - The decomposition of the complex measure $m$ into its real and imaginary parts is given by $m=(1 / 2(m+\bar{m}))+$ $i((1 / 2 i)(m-\bar{m}))=(\operatorname{Re} m)+i(\operatorname{Im} m)$ where $\bar{m}$ is defined as usual by $\int \bar{g} d m=\int \bar{g} d m$ for continuous functions $g$ on $T$. If the representable function $F \in P_{1}$ is given by $F=\int P_{C} d m+k$, then $G_{F}=$ $\int P_{C} d(\operatorname{Re} m)+1 / 2(k-\bar{k})$ and $H_{F}=\int P_{C} d(\operatorname{Im} m)+(1 / 2 i)(k+\bar{k}) .-$ If $m \in M$, we write $\hat{m}_{j}=\int e^{-i j t} d m$. 
The following theorem characterizes the elements of $P_{1}$ among those of $P$.

THEOREM 1. The function $F \in P$ is representable if and only if there is a constant $B_{F}$ such that

$$
\int\left|F\left(r e^{i t}\right)-F\left(r^{-1} e^{i t}\right)\right| d t \leqq B_{F} \text { for all } r \in[0,1) .
$$

Note that if $F$ is of real type this becomes Nevanlinna's condition $\int\left|\operatorname{Re} F\left(r e^{i t}\right)\right| d t \leqq B_{F}$ for all $r \in[0,1)$; we deduce our theorem from Nevanlinna's.

Proof. The representability of $F$ implying that of $G$ and $H$, Nevanlinna's theorem asserts the existence of constants $B_{G}$ and $B_{H}$ such that

$$
\int\left|\operatorname{Re} G\left(r e^{i t}\right)\right| d t \leqq B_{G}, \int\left|\operatorname{Re} H\left(r e^{i t}\right)\right| d t \leqq B_{H}
$$

for all $r \in[0,1)$. Thus, since $2 \operatorname{Re} G(z)=\operatorname{Re} F(z)-\operatorname{Re} F\left(\bar{z}^{-1}\right)$ and $2 \operatorname{Re} H(z)=\operatorname{Im} F(z)-\operatorname{Im} F\left(\bar{z}^{-1}\right)$, (3) implies (2). Conversely, let $F$ satisfy (2). Then $G$ and $H$ given by (1) satisfy (3): $1 / 2 \int\left|\operatorname{Re} F\left(r e^{i t}\right)-\operatorname{Re} F\left(r^{-1} e^{i t}\right)\right| d t=\int\left|\operatorname{Re} G\left(r e^{i t}\right)\right| d t \leqq 1 / 2 \int \mid F\left(r e^{i t}\right)-$ $F\left(r^{-1} e^{i t}\right) \mid d t \leqq B_{F}$ and similarly $1 / 2 \int\left|\operatorname{Im} F\left(r e^{i t}\right)-\operatorname{Im} F\left(r^{-1} e^{i t}\right)\right| d t=$ $\int\left|\operatorname{Re} H\left(r e^{i t}\right)\right| d t \leqq B_{F}$ so that by Nevanlinna's theorem there exist measures $m_{1}$ and $m_{2}$ in $M$ which are real such that $G=\int P_{C} d m_{1}+k_{1}$ and $H=\int P_{C} d m_{2}+k_{2}$ (for suitable constants $k_{1}, k_{2}$ ) so that $F=$ $\int P_{C}\left(d m_{1}+i d m_{2}\right)+\left(k_{1}+i k_{2}\right)=\int P_{C} d m+k$ with $m=m_{1}+i m_{2}, k=k_{1}+i k_{2}$ and $F \in P_{1}$.

Representations $F=\int P_{C} d m+k$ are clearly not unique: adding a multiple $a L$ of Lebesgue measure $(a \in C)$ to $m$ merely changes the constant: $\quad F=\int P_{C} d(m+a L)+k^{\prime}$ with $k^{\prime}=k-2 \pi a$ in $D$ and $k^{\prime}=$ $k+2 \pi a$ in $D^{c}$. It is, however, possible to standardize, and thus to make unique, the representations. This is done in the following theorem which also presents an inversion formula expressing $m$ and $k$ in terms of $F$.

THEOREM 2. If $F=\int P_{C} d m_{1}+k_{1}=\int P_{C} d m_{2}+k_{2}$ with $k_{1}$ the same constant in $D$ and in $D^{\circ}$ and similarly for $k_{2}$, then $m_{1}=m_{2}$ and $k_{1}=k_{2}$. If $F \in P_{1}$ and if we define

$$
m_{F}(t)=(1 / 4 \pi) \lim _{r \uparrow 1} \int_{0}^{t}\left(F\left(r e^{i s}\right)-F\left(r^{-1} e^{i s}\right)\right) d s
$$




$$
k_{F}=\frac{1}{2}(F(0)+F(\infty)) \text { both in } D \text { and } D^{c} \text {, }
$$

then

$$
F=\int P_{C} d m_{F}+k_{F}
$$

uniquely.

Thus all functions $F \in P_{1}$ have a (unique) representation with the constant the same in $D$ and $D^{c}$. We do not wish to confine ourselves to this representation in view of Theorems 4-7 below.

Proof. If $F=G+i H$ as in (1), then Nevanlinna's theory says that there are measures $m_{G}$ and $m_{H}$ and constants $k_{G}$ and $k_{H}$ given by $m_{G}(t)=(1 / 2 \pi) \lim _{r \uparrow 1} \int_{0}^{t} \operatorname{Re} G\left(r e^{i s}\right) d s=(1 / 4 \pi) \lim _{r \uparrow 1} \int_{0}^{t}\left(\operatorname{Re} F\left(r e^{i s}\right)-\right.$ $\left.\operatorname{Re} F\left(r^{-1} e^{i s}\right)\right) d s, G=\int P_{C} d m_{G}+k_{G}$ with $k_{G}=1 / 2(G(0)+G(\infty))=1 / 2(G(0)-$ $\overline{G(0)})=i \operatorname{Im} G(0)$ with similar expressions for $m_{H}, k_{H}, H$. Thus $m_{F}$ and $k_{F}$ are as given in (4) and (5) is therefore true. The uniqueness results from this: If $F=\int P_{C} d m+k$ where $k$ is the same constant in $D$ and $D^{c}$, then $F(0)=m(T)+k, F(\infty)=-m(T)+k$ so that $2 k=F(0)+F(\infty)$. If now $F=\int P_{c} d m_{1}+k=\int P_{C} d m_{2}+k$, then $\int P_{C} d m=0$ where $m=m_{1}-m_{2}$ so that $\hat{m}_{j}=0$ for all integers $j$ and $m=0, m_{1}=m_{2}$. - Note that (4) and (5) can also be deduced directly from our hypothesis (2) and the expressions

$$
\begin{aligned}
& F(z)=(1 / 4 \pi) \int\left(e^{i t}+r^{-1} z\right) /\left(e^{i t}-r^{-1} z\right)\left(F\left(r e^{i t}\right)-F\left(r^{-1} e^{i t}\right)\right) d t+k_{F}(|z|<r<1) \\
& F(z)=(1 / 4 \pi) \int\left(e^{i t}+r z\right) /\left(e^{i t}-r z\right)\left(F\left(r e^{i t}\right)-F\left(r^{-1} e^{i t}\right)\right) d t+k_{F}\left(|z|>r^{-1}>1\right) .
\end{aligned}
$$

Condition (2) which characterizes representability can be used to introduce a natural norm in $P_{1}$. If $F \in P_{1}$ define $\|F\|_{0}=$ $\sup _{0 \leqq r<1} \int\left|F\left(r e^{i t}\right)-F\left(r^{-1} e^{i t}\right)\right| d t$ and $\|F\|=\|F\|_{0}+\left|k_{F}\right|$. The following lemma relates $\|F\|_{0}$ to $\left\|m_{F}\right\|$ for $m_{F} \in M$.

LEMMA. $\|F\|_{0} \leqq 24 \pi\left\|m_{F}\right\| \leqq 6\|F\|_{0}$.

Proof. (1) We have for $m \in M$ the definition $\|m\|=\left.\operatorname{Var}\right|_{0} ^{2 \pi}[m]=$ $\sup _{E} \sum_{K}\left|m\left(t_{k}\right)-m\left(t_{k-1}\right)\right|$ over all partitions $E: 0=t_{0}<t_{1}<\cdots<t_{n}=2 \pi$ with $I_{k}=\left[t_{k-1}, t_{k}\right]$. Let $D_{F}(r, s)=D(r, s)=F\left(r e^{i s}\right)-F\left(r^{-1} e^{i s}\right)$. Then $\left\|m_{F}\right\|=(1 / 4 \pi) \sup _{E} \lim _{r} \sum_{k}\left|\int_{I_{k}} D(r, s) d s\right|$ where we have used (4). Thus $\left\|m_{F}\right\| \leqq(1 / 4 \pi) \sup _{E} \lim _{r} \sum_{k} \int_{I_{k}}|D(r, s)| d s=(1 / 4 \pi) \lim _{r} \int|D(r, s)| d s=$ $(1 / 4 \pi)\|F\|_{0}$. 
(2) When $F_{1}$ and $F_{2}$ are in $P_{1}$ we have $\left\|F_{1}+F_{2}\right\|_{0} \leqq\left\|F_{1}\right\|_{0}+$ $\left\|F_{2}\right\|_{0}$ since

$$
\begin{gathered}
\left|F_{1}+F_{2} \|_{0}=\sup _{0 \leqq r<1} \int\right| D_{F_{1}}+D_{F_{2}}\left|\leqq \sup \int\right| D_{F_{1}} \mid \\
+\sup \int\left|D_{F_{2}}\right|=\left\|F_{1}\right\|_{0}+\left\|F_{2}\right\|_{0} .
\end{gathered}
$$

Thus if $F=G+i H$ as in (1), we have $\|F\|_{0} \leqq\|G\|_{0}+\|H\|_{0}$ and since $2 m_{G}=m_{F}+\bar{m}_{F}$ and $2 i m_{H}=m_{F}-\bar{m}_{F}$ we have $\left\|m_{G}\right\| \leqq\left\|m_{F}\right\|$ and $\left\|m_{H}\right\| \leqq\left\|m_{F}\right\|$. We next establish the inequality $\|G\|_{0} \leqq 12 \pi\left\|m_{G}\right\|$. We have $G=G_{1}-G_{2}$ corresponding to a decomposition $m_{G}=m_{1}-m_{2}$ for positive measures $m_{1}$ and $m_{2}$. We also have $\|G\|_{0} \leqq\left\|G_{1}\right\|_{0}+\left\|G_{2}\right\|_{0}$. If some function $F_{0} \in P_{1}$ has nonnegative real part and so corresponds to a positive measure $m_{0}$, we have $\left\|F_{0}\right\|_{0}=2 \lim _{r} \int \operatorname{Re} F_{0}\left(r e^{i t}\right) d t=$ $4 \pi \operatorname{Re} F_{0}(0)=4 \pi m_{0}(T)=4 \pi\left\|m_{0}\right\|$. Let now $m_{1}(t)=\left.\operatorname{Var}\right|_{0} ^{t}\left[m_{G}\right]$ and $m_{2}(t)=m_{1}(t)-m_{G}(t)$. Then $\left\|m_{1}\right\|=\left\|m_{G}\right\|$ and $\left\|m_{2}\right\| \leqq 2\left\|m_{G}\right\|$ so that $\|G\|_{0} \leqq\left\|G_{1}\right\|_{0}+\left\|G_{2}\right\|_{0} \leqq 12 \pi\left\|m_{G}\right\| \leqq 12 \pi\left\|m_{F}\right\|$ and similarly $\|H\|_{0} \leqq$ $12 \pi\left\|m_{F}\right\|$, i.e., the first inequality asserted in the lemma is proved.

THEOREM 3. The function $F \mapsto\|F\|=\|F\|_{0}+\left|k_{F}\right|$ is a norm on $P_{1}$. The map $\phi: M \times C \rightarrow P_{1}$ given by $(m, k) \mapsto F=\int P_{c} d m+k$ is a 1-1 linear bicontinuous map of the Banach space $M \times C$ (with usual norm topology) onto $P_{1}$ (relative to the norm topology based on $\|F\|)$ so that, in particular, $P_{1}$ is a Banach space with its norm. A sequence $\left(m_{j}, k_{j}\right)$ converges to $\left(m_{0}, k_{0}\right)$ where the convergence of the measures is weak* and that of the $k_{j}$ the ordinary convergence of complex numbers if and only if $F_{j} \rightarrow F_{0}$ for the corresponding functions uniformly on compact sets and there exists a constant $B$ with $\left\|F_{j}\right\| \leqq B$ for all positive $j$.

Note that $\phi$ would not be well-defined if we did not use the (unique) representation of $F$ with $k$ the same in $D$ and $D^{c}$; see (4), (5).

Proof. (1) The first part of the theorem is just a summary of assertions proved earlier. (2) Suppose $m_{j} \rightarrow m_{0}$ weak*. Then $\int P_{C} d m_{j} \rightarrow$ $\int P_{c} d m_{0}$ pointwise in $D \cup D^{c}$; on every compact subset of $D \cup D^{c}$ the family $\left\{\int P_{C} d m_{j}\right\}$ is uniformly bounded so that by virtue of normal family theory the convergence $\int P_{C} d m_{j} \rightarrow \int P_{C} d m_{0}$ is uniform on compact sets. The weak* convergence of $m_{j}$ to $m_{0}$ says that the $\left\|m_{j}\right\|$ and hence, by the lemma, the $\left\|F_{j}\right\|_{0}$ are bounded. The convergence $\int P_{C} d m_{j} \rightarrow \int P_{C} d m_{0}$ uniformly on compacts and the convergence $k_{j} \rightarrow k_{0}$ 
imply that $F_{j} \rightarrow F_{0}$ uniformly on compacts and that the $\left\|F_{j}\right\|$ are bounded.-The converse is similar: If $F_{j} \rightarrow F_{0}$ uniformly on compacts and if the $\left\|F_{j}\right\|$ are bounded, then first by the lemma the $\left\|m_{j}\right\|$ are bounded and $\int t d m_{j} \rightarrow \int t d m_{0}$ on the dense subset $\{t\}$ of trigonometric polynomials on $T$ and this, together with the boundedness of the $\left\|m_{j}\right\|$ implies the weak* convergence of $m_{j}$ to $m_{0}$.

Note that if we consider the restriction $\phi_{r}$ of $\phi$ to $M$, then the image $\phi(M)$ in $P_{1}$ is the closed subspace consisting of all $F$ with $F(0)+F(\infty)=0$. Then map $\phi_{r}$ has of course the same properties as $\phi$.

Let $F(D)$ and $F\left(D^{c}\right)$ be the parts of $F \in P$ in $D$ and $D^{\circ}$ respectively. When $F$ is merely in $P$, the relation between $F(D)$ and $F\left(D^{c}\right)$ is of course totally arbitrary. If, however, $F \in P_{1}$, there is a relation. First, if we take two arbitrary functions $f$ and $g$ with the proviso that $f$ be holomorphic in $\{z ;|z|<1+a\}$ and $g$ holomorphic in $\{z ;|z|>1-b\}$ (for positive $a$ and $b$ ) and then combine their restrictions to $D$ and $D^{\circ}$ respectively, then $F$ with $F(D)=f$ and $F\left(D^{c}\right)=g$ will be in $P_{1}$; otherwise, however, the relation between $F(D)$ and $F\left(D^{c}\right)$ is governed by the following theorems.

THEOREM 4. A function $F \in P$ with $F(D)$ constant is in $P_{1}$ if and only if $F=\int P_{C} d m+k$ where $m$ is absolutely continuous with derivative $f_{T} \in L_{1}$ and Fourier series $\sum_{0} e^{-i j t} a_{j}$ and which is the boundary function of $f(z)$ antiholomorphic in $D$ given by $\sum_{0} \bar{z}^{j} a_{j}$. Similarly $F\left(D^{c}\right)$ is constant if and only if $F=\int P_{C} d m+k$ with absolutely continuous $m$ whose derivative $g_{T} \in L_{1}$ has Fourier series $\sum_{0} e^{i j t} b_{j}$ and is the boundary function of $g(z)=\sum_{0} z^{j} b_{j}$ holomorphic in $D$.

Proof. This is just the F. and M. Riesz theorem-the necessities are obvious. Suppose now that $\int P_{c} d m+k=d$, a constant in $D$, then $m(T)+k=d$ and, since $P_{C}=1+2 \sum_{1} e^{-i j t} z^{j}$ in $D$, we conclude that $\int P_{C} d m+k=\int\left(1+2 \sum_{1} e^{i j t} z^{j}\right) d m+k=d+2 \sum_{1} \hat{m}_{j} z^{j}=d$ so that $\hat{m}_{j}=0=\int e^{-i j t} d m(j=1, \cdots)$ i.e., $\int e^{i j t} d \bar{m}=0$ for all positive integers $j$, so that $\bar{m}$ is absolutely continuous with derivative $\bar{m}^{\prime}=\bar{f}_{T}(t)$ with Fourier series $\sum_{0} e^{i j t} \bar{a}_{j}$ and the first half of the theorem is proved. The second half proceeds the same way.

Let $M_{0}$ be the subset of $M$ consisting of absolutely continuous measures with derivatives $g_{T} \in L_{1}$ with Fourier series $\sum_{1} a_{j} e^{-i j t}$.

THEOREM 5. A function $f$ holomorphic in $D$ is the part $F(D)$ for some function $F \in P_{1}$ if and only if $\operatorname{dist}_{0 \leqq r<1}\left(m_{r}, M_{0}\right) \leqq B<\infty$ for 
some constant $B$ where $m_{r}$ is the absolutely continuous measure with derivative $f\left(r e^{i t}\right)$ and dist is based on the usual norm in $M$.

This criterion contains the criteria contained in the HerglotzRiesz and Nevanlinna theorems.

Proof. For the necessity suppose we have $F \in P_{1}$ with $F(D)=f$ in $D$. To show that $\operatorname{dist}_{0 \leqq r<1}\left(m_{r}, M_{0}\right) \leqq B$, we find for each $r \in[0,1)$ a measure $n_{r} \in M_{0}$ with $\left\|m_{r}-n_{r}\right\|=\sup _{\|c\|_{\infty}=1}\left|\int c d\left(m_{r}-n_{r}\right)\right| \leqq B$, taken over all continuous functions $c$ on $T$. Now $m_{r}^{\prime}=f\left(r e^{i t}\right)=F(D)\left(r e^{i t}\right)=$ $F\left(r e^{i t}\right)$ and our basic criterion (2) furnishes $n_{r}$ with $n_{r}^{\prime}=F\left(D^{c}\right)\left(r^{-1} e^{i t}\right)=$ $F\left(r^{-1} e^{i t}\right)$ so that $\operatorname{dist}\left(m_{r}, M_{0}\right) \leqq\left\|m_{r}-n_{r}\right\| \leqq \sup _{r} \int\left|F\left(r e^{i t}\right)-F\left(r^{-1} e^{i t}\right)\right| d t \leqq$ $B<\infty$.

For the sufficiency, suppose that $\operatorname{dist}\left(m_{r}, M_{0}\right) \leqq B$. This implies by the weak* compactness of bounded sets in $M$ that there exists a sequence $r_{j} \in[0,1)$ with $r_{j}$ increasing to 1 and measures $n_{r} \in M_{0}$ such that $m_{r}-n_{r} \rightarrow m \in M$ (weak* convergence). Write $n_{r}^{\prime}=\sum_{1} a_{j}(r) e^{-i j t}$. Then $L(r)=\int P_{C}\left(f\left(r e^{i t}\right)-\sum_{1} a_{j}(r) e^{-i j t}\right) d t \rightarrow \int P_{C} d m$ as $r \uparrow 1$. Now $L(r)=$ $4 \pi f(r z)-2 \pi f(0)$ while $\int P_{C} d m$ furnishes a function (in $D^{c}$ ) that is holomorphic in $D^{c}$. Thus $\int P_{c} d m=F \in P_{1}$ yields a function with $F(D)=f+$ const.

It is clear from this argument and from Theorem 4 that there are many functions $F \in P_{1}$ with $F(D)=f+$ const.: the difference of any two of them is characterized in the second half of that theorem.

In addition to its Banach space structure, $M$ has also a ring structure with respect to convolution of measures. The corresponding ring structure in $P_{1}$ is given by the Hadamard product: If $f$ and $g$ have expansions $\sum_{0} a_{j} z^{j}$ and $\sum_{0} b_{j} z^{j}$ respectively, define $f^{*} g$ by $\sum_{0} a_{j} b_{j} z^{j}$, if $f$ and $g$ have expansions $\sum_{0} c_{j} z^{-j}$ and $\sum_{0} d_{j} z^{-j}$ respectively, define $f^{*} g$ by $-\sum_{0} c_{j} d_{j} z^{-j}$. If $F$ and $G$ are in $P$, then the Hadamard product $F^{*} G$ is defined in $D$ and $D^{\circ}$ according to the rules just mentioned for $D$ and $D^{c}$ respectively.

THEOREM 6. If $F_{1}$ and $F_{2}$ are in $P_{1}$ with $F_{j}=\int P_{C} d m_{j}+k_{j}$ where the $k_{j}$ are piecewise constants in $P_{1}$ then $F_{1}{ }^{*} F_{2}=F=\int P_{c} d m+k \in P_{1}$ and

$$
m=2\left(m_{1}^{*} m_{2}\right), k=\begin{aligned}
& -m_{1}(T) m_{2}(T)+k_{1} k_{2}+k_{1} m_{2}(T)+k_{2} m_{1}(T) \\
& \\
& +m_{1}(T) m_{2}(T)-k_{1} k_{2}+k_{1} m_{2}(T)+k_{2} m_{1}(T)
\end{aligned}
$$

in $D$ and $D^{\circ}$ respectively. 
Proof. The proof is a simple calculation based on the formula $\left(m_{1}{ }^{*} m_{2}\right)_{j}=\hat{m}_{1, j} \hat{m}_{2, j}: \quad$ If $z \in D$ then $\int P_{C} d\left(m_{1}{ }^{*} m_{2}\right)=m_{1}(T) m_{2}(T)+$ $2 \sum_{1} \hat{m}_{1, a} \hat{m}_{2, a} z^{a}$ with analogous expansions for $F_{1}$ and $F_{2}$. We obtain $\left(F_{1}{ }^{*} F_{2}\right)(z)=\left(m_{1}(T)+k_{1}\right)\left(m_{2}(T)+k_{2}\right)+4 \sum_{1} \hat{m}_{1, a} \hat{m}_{2, a} z^{a}$ in $D$ with a similar equation in $D^{c}$ so that (7) is established.

Corollary. The map $(F, G) \mapsto F * G$ is continuous in both variables in the norm of $P_{1}$.

The usual Banach algebra inequality $\|F * G\| \leqq\|F\|\|G\|$ is not valid in $P_{1}$ : take $F=G=10+z$ in $D$ and equal to $10+z^{-1}$ in $D^{c}$. The map $\phi$ of Theorem 3 is thus not an isometry.

- If $m \in M$, define $F_{m}$ by

$$
F_{m}=\frac{1}{2} \int P_{C} d m+k_{m}, 2 k_{m}=-m(T) \text { in } D \text { and } m(T) \text { in } D^{c} .
$$

Let $P_{2}$ be the subset of $P_{1}$ consisting of all $F$ will $F(0)=F(\infty)=0$; it is a closed subalgebra of $P_{1}$. The following immediate consequence of the preceding theorem is worth stating separately.

THEOREM 7. The map $\psi: M \rightarrow P_{2}$ given by (8) is a linear continuous open epimorphism of the Banach algebra $M$ to the Banach algebra $P_{2}$ with kernel the constant multiples of Lebesgue measure.

Similar statements are valid about other subalgebras of $M \times \mathbf{C}$ and $P_{1}$, e.g., for the subalgebra of $M$ of all $m$ with $m(T)=0$ and the subalgeba of $P_{2}$ of all $F$ with $k_{F}=0$; the kernel of the restriction of $\psi$ to this subalgebra of $M$ is determined on the basis of Theorem 4.

Our using complex measures makes the following considerations possible. We define derivatives of functions in $P$ as usual (i.e., in $D$ and $D^{\circ}$ separately). If $G=F^{\prime}$ for functions in $P$ we call $F$ an integral of $G$. We shall use the phrase that $F$ is differentiable (or integrable) in $P_{1}$ if $F$ and $F^{\prime}$ are in $P_{1}$. Differentiability of $F$ in $P_{1}$ imposes a strong restriction of $F$; integrability is much less restrictive although infinite integrability is of course very restrictive. In what follows all functions in $P_{1}$ will be in the standard representation (5).

THEOREM 8. A function $F=\int P_{c} d n+k$ has a derivative $F^{\prime}=$ $\int P_{C} d m+k^{\prime}$ (all in $P_{1}$ ) if and only if $n$ is absolutely continuous with derivative $g$ of bounded variation and $g(0)=g(2 \pi)=0$. If $F$ 
is differentiable in $P_{1}$ then $m(t)=-i \int_{0}^{t} e^{-i s} d g, \hat{m}_{-1}=0, k^{\prime}=m(T)$. Or equally well: A function $G=\int P_{C} d m+k^{\prime}$ has an integral $F=$ $\int P_{c} d n+k\left(\right.$ all in $P_{1}$ ) if and only if $\hat{m}_{-1}=0$ and $k^{\prime}=m(T)$. If $G$ is integrable in $P_{1}$ then $n$ is absolutely continuous with derivative $g$ of bounded variation and $g(0)=g(2 \pi)=0$, and $g(t)=i \int_{0}^{t} e^{i s} d m$.

Proof. We prove the first version of the theorem. Necessity: (1) If $F^{\prime}=\int\left(-1-2 \sum_{1} z^{-j} e^{i j t}\right) d m(t)+k^{\prime}=-\hat{m}_{0}-2 \sum_{1} \hat{m}_{-j} z^{j}+k^{\prime}$ (expansion in $D^{c}$ ) is to be the derivative of a function in $P_{1}$, we must have $-\hat{m}_{0}+k^{\prime}=-m(T)+k^{\prime}=0$ and $\hat{m}_{-1}=0$.

(2) Consider $i \int P_{C} \int_{0}^{t} e^{i s} d m(s) d t$ and expand $P_{C}$. Treat $D$ and $D^{c}$ separately. In the expansion, change the order of integration and differentiate; using $\hat{m}_{-1}=0$ and $k^{\prime}=m(T)$, we see that we have obtained $F^{\prime}$. Thus $F=\int P_{C} d n+k=i \int P_{C} \int_{0}^{t} e^{i s} d m(s) d t+$ const.; the uniqueness assertion of Theorem 2 then implies that $n$ is absolutely continuous whose derivative $g(t)=i \int_{0}^{t} e^{i s} d m(s)$ which is of bounded variation with $g(0)=0$ and $g(2 \pi)=i \hat{m}_{-1}=0$; this also shows that $m(t)=$ $-i \int_{0}^{t} e^{-i s} d g(s)$ as desired.-Sufficiency: Suppose $F=\int P_{C} d n+k$ with absolutely continuous $n$ whose derivative $g$ is of bounded variation and $g(0)=g(2 \pi)=0$ : to show that $F$ is differentiable in $P_{1}$ with $F^{\prime}=\int_{c} P_{c} d m+m(T)$ where $m(t)=-i \int_{0}^{t} e^{-i s} d g(s)$ and $\hat{m}_{-1}=0$; the last equation is immediate: $\hat{m}_{-1}=g(2 \pi)-g(0)=0$. Consider now $\int P_{C} d m+m(T)$ where $m$ is defined as above. Again we proceed by expanding $P_{C}$ and treat $D$ and $D^{\circ}$ separately. Thus $\int P_{C} d m+m(T)=$ $-i \int P_{c} e^{-i t} d g(t)-i \int e^{-i t} d g(t)$. After expanding, we integrate by parts and observe that we have obtained $F^{\prime}$ as expected.

This completes the proof of the theorem.

The second part of the following corollary is again a result of the F. and M. Riesz theorem on analytic measures.

CoRollary. A function $F=\int P_{C} d m$ is infinitely differentiable in $P_{1}$ if and only if $m \in C_{\infty}$ and $m^{(j)}(0)=m^{(j)}(2 \pi)=0$ for all positive integers $j$. A function in $P_{1}$ is infinitely integrable in $P_{1}$ if and only if it is zero in $D^{\circ}$.

The preceding results can all be phrased in terms of FourierStieltjes moments. We single out the following application. It is 
clear that if $\left\{j n_{j}\right\}$ is a moment sequence corresponding to the measure $m$, then $\left\{n_{j}\right\}$ is also a moment sequence whose measure $n$ is absolutely continuous with derivative of bounded variation $i m$. This can also be read from Theorem 8; the hypothesis in the necessity part of that theorem which says that $F$ be in $P_{1}$ can be replaced by demanding merely that $F \in P$. We consider in the following theorem a certain kind of perturbation of the multiplier sequence $\{j\}$ of $\left\{j n_{j}\right\}$; we obtain the same conclusion as for that latter sequence.

THEOREM 9. If $\left\{a_{j} n_{j}\right\}$ is a moment sequence and if the analytic functions $\sum_{1}\left(j-a_{j}\right) z^{j}$ and $\sum_{1}\left(j+a_{-j}\right) z^{j}$ have radii of convergence greater than 1 , then $\left\{n_{j}\right\}$ is a moment sequence corresponding to an absolutely continuous measure whose derivative is of bounded variation.

Proof. We note that the function $F$ defined in $D$ by $\sum_{1} a_{j} n_{j} z^{j}$ and in $D^{\circ}$ by $-\sum_{1} a_{-j} n_{-j} z^{-j}$ is in $P_{1}$ since $\left\{a_{j} n_{j}\right\}$ is a moment sequence by hypothesis. We shall show that $\left\{j n_{j}\right\}$ is a moment sequence; we show first that the function $G$ defined in $D$ by $\sum_{1} j n_{j} z^{j}$ and in $D^{\circ}$ by $\sum_{1} j n_{-j} z^{-j}$ is in $P_{1}$; we use the criterion (2) of Theorem 1 . We have

$$
\begin{aligned}
G\left(r e^{i t}\right)-G\left(r^{-1} e^{i t}\right)= & \sum_{1} r^{j}\left(j n_{j} e^{i j t}-j n_{-j} e^{-i j t}\right) \\
= & \sum_{1} r^{j}\left[\left(j-a_{j}\right) n_{j} e^{i j t}-\left(j+a_{-j}\right) n_{-j} e^{-i j t}\right] \\
& +\sum_{1} r^{j}\left(a_{j} n_{j} e^{i j t}+a_{-j} n_{-j} e^{-i j t}\right) .
\end{aligned}
$$

We show next that the sequence $\left\{n_{j}\right\}$ is bounded: since $\left\{a_{j} n_{j}\right\}$ is a moment sequence, it is bounded, say, $\left|a_{j} n_{j}\right| \leqq B$; since the power series mentioned in the statement of the theorem have radii of convergence greater than 1 , we will have for sufficently large $j$ the inequalities $\left|j-a_{j}\right| \leqq 1$ and $\left|j+a_{-j}\right| \leqq 1$ so that $\left|a_{j}\right| \geqq|j|-1$ whence $\left|n_{j}\right| \leqq B$ as desired. We now take absolute values in (9) and integrate with respect to $t$. Thus

$$
\begin{array}{r}
\int\left|G\left(r e^{i t}\right)-G\left(r^{-1} e^{i t}\right)\right| d t \leqq \int\left|f\left(r e^{i t}\right)-\overline{g\left(r e^{i t}\right)}\right| d t \\
\quad+\int\left|F\left(r e^{i t}\right)-F\left(r^{-1} e^{i t}\right)\right| d t=T_{1}(r)+T_{2}(r)
\end{array}
$$

where $f$ and $g$ are the analytic functions with radii of convergence greater than 1 mentioned in the theorem. Thus $T_{1}(r) \leqq B_{1}$ for all $r \in(0,1)$ and $T_{2}(r) \leqq B_{2}$ since $F \in P_{1}$. Thus $G \in P_{1},\left\{j n_{j}\right\}$ is a moment sequence and the theorem is proved.

Analogous problems for several variables and also for regions 
other than $D$ and $D^{c}$ such as complementary half planes will be dealt with in another paper.

Received November 1, 1976 and in revised form March 23, 1977.

UNIVERSITY OF CALIFORNIA

IRVINE, CA 92664 


\title{
PACIFIC JOURNAL OF MATHEMATICS
}

\author{
EDITORS
}

RICHARD ARENS (Managing Editor)

University of California

Los Angeles, California 90024

C. W. CurTis

University of Oregon

Eugene, OR 97403

C. C. MOORE

University of California

Berkeley, CA 94720
J. DUgundJI

Department of Mathematics

University of Southern Californı

Los Angeles, California 90007

R. Finn and J. Milgram

Stanford University

Stanford, California 94305

\section{ASSOCIATE EDITORS}

E. F. BECKENBACH

\section{SUPPORTING INSTITUTIONS}

\author{
UNIVERSITY OF BRITISH COLUMBIA \\ CALIFORNIA INSTITUTE OF TECHNOLOGY \\ UNIVERSITY OF CALIFORNIA \\ MONTANA STATE UNIVERSITY \\ UNIVERSITY OF NEVADA, RENO \\ NEW MEXICO STATE UNIVERSITY \\ OREGON STATE UNIVERSITY \\ UNIVERSITY OF OREGON \\ OSAKA UNIVERSITY
}

UNIVERSITY OF SOUTHERN CALIFORNIA

STANFORD UNIVERSITY

UNIVERSITY OF TOKYO

UNIVERSITY OF UTAH

WASHINGTON STATE UNIVERSITY

UNIVERSITY OF WASHINGTON
AMERICAN MATHEMATICAL SOCIETY
NAVAL WEAPONS CENTER 


\section{Pacific Journal of Mathematics \\ Vol. 72 , No. 1 \\ January, 1977}

Kazuo Anzai and Shiro Ishikawa, On common fixed points for several

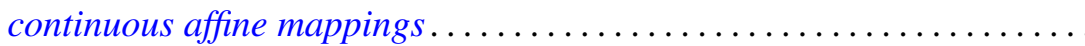

Bruce Alan Barnes, When is a representation of a Banach $*$-algebra

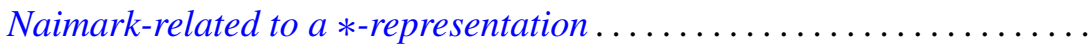

Richard Dowell Byrd, Justin Thomas Lloyd, Franklin D. Pedersen and

James Wilson Stepp, Automorphisms of the semigroup of finite

complexes of a periodic locally cyclic group ...................

Donald S. Coram and Paul Frazier Duvall, Jr., Approximate fibrations and a

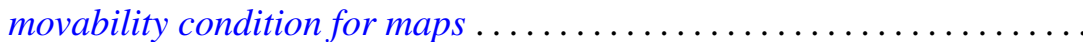

Kenneth R. Davidson and Che-Kao Fong, An operator algebra which is not

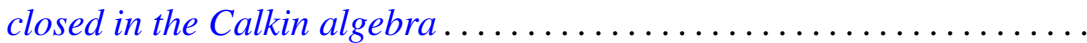

Garret J. Etgen and James Pawlowski, A comparison theorem and oscillation criteria for second order differential systems .............

Philip Palmer Green, $C^{*}$-algebras of transformation groups with smooth

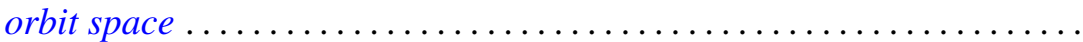

Charles Allen Jones and Charles Dwight Lahr, Weak and norm approximate

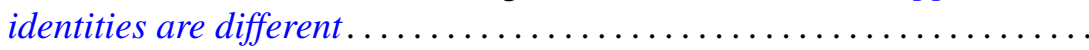

G. K. Kalisch, On integral representations of piecewise holomorphic

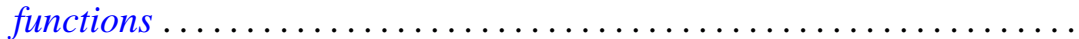

Y. Kodama, On product of shape and a question of Sher

Heinz K. Langer and B. Textorius, On generalized resolvents and

$Q$-functions of symmetric linear relations (subspaces) in Hilbert

space ...................................

Albert Edward Livingston, On the integral means of univalent, meromorphic functions

Wallace Smith Martindale, III and Susan Montgomery, Fixed elements of

Jordan automorphisms of associative rings ..........

R. Kent Nagle, Monotonicity and alternative methods for nonlinear boundary value problems ........................

Richard John O'Malley, Approximately differentiable functions: the $r$ topology.

Mangesh Bhalchandra Rege and Kalathoor Varadarajan, Chain conditions

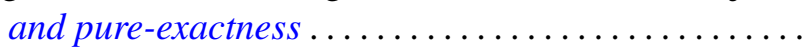

Christine Ann Shannon, The second dual of $C(X)$. .

Sin-ei Takahasi, A characterization for compact central double centralizers of $C^{*}$-algebras .

Theresa Phillips Vaughan, A note on the Jacobi-Perron algorithm. . .

Arthur Anthony Yanushka, A characterization of $\operatorname{PSp}(2 m, q)$ and

$\mathrm{P} \Omega(2 m+1, q)$ as rank 3 permutation groups ......... 